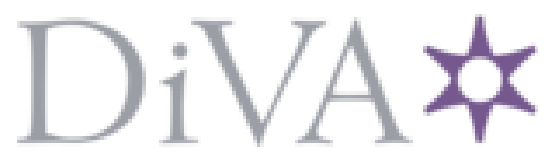

http://www.diva-portal.org

This is the published version of a paper published in International journal of sports science \& coaching.

Citation for the original published paper (version of record):

Ryman Augustsson, S., Jesper, A., Thomeé, R., Karlsson, J., Eriksson, B. et al. (2011)

Performance Enhancement Following a Strength and Injury Prevention Program: A 26-Week

Individualized and Supervised Intervention in Adolescent Female Volleyball Players.

International journal of sports science \& coaching, 6(3): 399-417

Access to the published version may require subscription.

N.B. When citing this work, cite the original published paper.

Permanent link to this version:

http://urn.kb.se/resolve?urn=urn:nbn:se:Inu:diva-51282 


\title{
Performance Enhancement Following a Strength and Injury Prevention Program: A 26-Week Individualized and Supervised Intervention in Adolescent Female Volleyball Players
}

\author{
Sofia Ryman Augustsson',2, Jesper Augustsson 2,3, \\ Roland Thomeé ${ }^{2}$, Jon Karlsson ${ }^{2}$, Bengt I Eriksson ${ }^{2}$ and \\ Ulla Svantesson' \\ ${ }^{1}$ Institute of Neuroscience and Physiology/Physiotherapy, \\ University of Gothenburg, Sweden \\ ${ }^{2}$ Institute of Clinical Sciences, Lundberg Laboratory for Orthopaedic
}

Research, Department of Orthopaedics, Sahlgrenska University Hospital, University of Gothenburg, S-413 45 Göteborg, Sweden

E-mail: sofia.augustsson@gu.se

${ }^{3}$ Department of Food and Nutrition, and Sport Science, Centre for Health and Performance, University of Gothenburg, Sweden

\begin{abstract}
The aim of this study was to evaluate the effects of a 26 -week individualized and supervised strength and injury prevention program on performance enhancement in young female volleyball players who completed resistance training with either a supervised and individualized training program (experimental group, $\mathrm{n}=10$ ) or an unsupervised nonindividualized training program (control group, $n=17$ ). Exposure and injury data were collected during the 2006-2007 season (baseline season) and the 26-week program was conducted during the 2007-2008 season (intervention season). All players were tested for physical performance. At post-test, the players in the experimental group had improved significantly more than the players in the control group for squat $(p<0.0001)$, bench press $(p=0.048)$, push-ups $(p=0.02)$ and sit-ups $(p<0.0001)$ but not for the vertical jump test. Thirty-five percent $(6 / 17)$ of the players from the control group and $80 \%(8 / 10)$ of the players in the experimental group completed the resistance training with compliance of no less than $50 \%$. The present study shows the importance of individualization and supervision for resistance training in young female athletes when it comes to compliance, strength gains and performance.
\end{abstract}

Key words: Exercise Adherence, Injury Prevention, Periodisation of Training, Strength and Conditioning, Women's Volleyball

Reviewers: $\quad$ Mike Lambert (University of Cape Town, South Africa) Harvey Newton (Newton Sports, USA) 


\section{INTRODUCTION}

It is well known that adolescent and adult female athletes have a four to eight times higher incidence of sustaining a serious knee ligament injury, such as an anterior cruciate ligament (ACL) injury, compared with male athletes participating in the same sport ${ }^{1,2}$. Further, it has been noted that adolescent female athletes have a muscle strength development in the lower extremities that is only half that compared with adolescent males ${ }^{3}$. This weak muscle strength development may be one of several factors responsible for the gender disparity in knee ligament injury rates and indicates that future studies should examine the benefits of resistance training for injury prevention in young female athletes.

It has been reported that, although more than $90 \%$ of female and male volleyball players include injury prevention programs in ordinary sports training and resistance training in particular, one in two volleyball players sustain injuries ${ }^{4}$. Although the primary reason for resistance training is performance enhancement, it has also been used to prevent sport injuries $^{5,6}$. For example, resistance training has been recommended for treating and reducing the risk of volleyball-related injuries, such as rotator cuff injuries and jumper's knee ${ }^{7-10}$. In addition, muscle strength appears to have a significant impact on outcome and the ability to return to sport after anterior cruciate ligament surgery ${ }^{11}$. Hence, resistance training plays an important role in both injury prevention and rehabilitation. However, resistance training itself may not guarantee optimal achievements in strength and performance. The outcome is dependent on the individual effort and systematic structuring of the training program ${ }^{12}$. Although there is evidence to suggest that variables, such as intensity, volume, frequency and type of exercises, in training programs aiming at physical performance and injury prevention should be individualized ${ }^{12,13}$ programs are still non-individualized in sport-scientific intervention studies ${ }^{14,15}$. Moreover, in practice, it has been our observation that many strength and conditioning programs performed by team athletes are non-individualized. It has also been reported that direct supervision promotes the magnitude and rate of progression in strength training ${ }^{16}$. In addition, supervision may be important when it comes to keeping the programs safe ${ }^{12,17}$. However, even if training is supervised, it can still be inappropriate and harmful if the physical trainer is inexperienced in managing sport-specific injuries and/or in designing individual training programs ${ }^{12}$. For this reason, the presence of a person that has specific strength and conditioning skills (e.g., physical therapist, athletic trainer, personal trainer) can be significant when it comes to the preparation of the athlete. In particular, the strength and conditioning coach can provide support for the athlete in the form of technique analysis and modification, motivation, goal setting and physical strengthening during training ${ }^{18,19}$. However, while it appears to be generally accepted that a qualified strength and conditioning coach plays an important role in improving athletic performance, few studies have examined the influence of coaching or supervision on physical performance and injury prevention. To our knowledge, there is no previously published study that has examined the influence of supervision and individualization during one season of a resistance-training program in young female athletes. An individualized and supervised resistance-training program in female athletes may provide an insight into enhancing performance and preventing injury.

The aim of this study was to evaluate the effects of a 26-week individualized and supervised strength and injury prevention program on performance enhancement in young female volleyball players. The injury panorama was also investigated during two seasons, where the first was the baseline season and the second the intervention season. 


\section{METHOD}

\section{PARTICIPANTS}

Female volleyball players from four (of six) teams in the third division, Göteborg volleyball federation, Sweden, were invited to participate in the study. All four teams agreed to participate in the study and, stratified by team, were randomly placed into either an experimental group (two teams and a total of 20 players) or a control group (two teams and a total of 20 players). No differences were found between the groups in terms of age and playing level. None of the participants had earlier experience with resistance training within the teams. None of the teams had a physical coach, but all teams had previously performed conditioning training instructed by the main coach. Written information was given to each player and written informed consent was obtained. Ethical approval was received from the Human Ethics Committee at Gothenburg University, Sweden. After the baseline season, one of the teams ceased to exist and was consequently excluded from the study. This team dropped out before the start of the intervention season as they no longer played volleyball. As a result, one team was present as an experimental group $(n=13)$ and two teams as a control group $(n=20)$. The sample of players still provided a statistical power of 0.88 to detect a group difference of $50 \%$. We therefore elected to continue with the sample size of 33 players. The players and coaches were informed about the experimental procedures and the possible risks and benefits of the project. Players with an injury at the start of the study were included in the study, but the specific injury was excluded from the injury statistics. Table 1 gives the characteristics of the players who participated in the study.

Table 1 Players Characteristics by Group at the Pre-Test $(n=27)$ Values are means ( \pm standard deviation).

\begin{tabular}{lcc} 
& Experimental $(\mathbf{n}=\mathbf{1 0})$ & Control $(\mathbf{n}=\mathbf{1 7})$ \\
\hline Age $($ years $)$ & $18( \pm 2)$ & $16( \pm 2)$ \\
\hline Weight $(\mathrm{kg})$ & $66( \pm 10)$ & $60( \pm 4)$ \\
\hline Height $(\mathrm{cm})$ & $171( \pm 7)$ & $168( \pm 5)$
\end{tabular}

\section{PROCEDURE}

All the teams included in the study participated in a continuous prospective injury registration protocol during the study period, season 2006-2007 (baseline season), used as baseline data, and season 2007-2008 (intervention season). All the teams were tested for physical performance, on a first occasion (pre-test) during the pre-season in September 2007. The experimental group and one team from the control group were tested one week prior to the initiation of the intervention. The other team from the control group was tested three weeks prior to the start of the intervention. The intervention started after the pre-test and lasted during the 26-week intervention season, from October 2007 to March 2008. All the teams received a program of resistance training exercises that was designed by the first author and aimed to improve physical performance and prevent injuries (Table 2). This resistance training program was given in addition to the regular training routine for all teams (both control and experimental group). Baseline information relating to the players in the experimental group was collected at the beginning of the intervention season. This information included injuries that did not cause absence from practice or games. The pain level of these injuries was then estimated by the players using a $100 \mathrm{~mm}$ Visual Analog Scale (VAS), where 0 is no pain and 100 is the worst pain imaginable. The estimation was made three times during the intervention season, in September 2007, in January 2008 and in April 
2008. The result of the pain estimations was registered by the first author. This information was then used as a tool when designing the individualized training programs (e.g., players experiencing anterior knee pain were given neuromuscular exercises for the knee). The teams in the control group were reminded once a month during the intervention season to continue with the resistance training program. The coaches of the teams in the control group recorded compliance on a designated form as the number of resistance training sessions and the duration of each session in minutes. All the teams were tested on a second occasion (posttest) in April 2008, after the end of the intervention season.

Table 2. Basic Program (4 w) for the Players in the Experimental Group and the Control Group, Presented by Exercise, Number of Sets and Repetitions and Rest Periods Between Sets

\begin{tabular}{llll} 
Exercise & Sets & Repetitions & Rest (min) \\
\hline Squat & 3 & 15 & 3 \\
\hline Bench press* & 3 & 15 & 2 \\
\hline Push-ups* & 2 & $\max$ & 1 \\
\hline Lat pulldown & 3 & 15 & 1 \\
\hline Seated cable row & 3 & 15 & 1 \\
\hline Overhead dumbbell press** & 3 & 12 & 2 \\
\hline Machine overhead press** & 2 & 15 & 1 \\
\hline Bodyweight back extension & 3 & 15 & 1 \\
\hline Machine crunch & 3 & 15 & 1 \\
\hline Sit-ups & 2 & $\max$ & 1
\end{tabular}

* Exercises that were performed in supersets for the experimental group.

** Exercises that were alternated every second training session for the experimental group.

\section{TESTING SCHEDULE}

Five tests were used to assess the players' physical performance: vertical jump, 1 RM squat, $1 \mathrm{RM}$ bench press, push-ups and sit-ups. All the players received a test manual two weeks before the pre-test. The tests were performed in the same order on both test occasions. The sequence of the tests was: vertical jump, 1 RM squat, 1 RM bench press, push-ups and sit-ups. The teams were also tested at the same time of the day, in the morning or the afternoon. The players were given strong verbal encouragement to perform all the tests as well as they could. During the pre-test, height and weight were noted for each player and the weight was noted again at the post-test. Each test session began with a warm-up, which consisted of five minutes of ergometer cycling at $100 \mathrm{~W}$ of resistance. The vertical jump, $1 \mathrm{RM}$ squat and $1 \mathrm{RM}$ benchpress tests were instructed and supervised by three blinded test leaders, one for each test, who did not know the players' team or group affiliations. The push-up and sit-up tests were instructed and supervised by a non-blinded test leader (the first author). These tests were chosen as they were considered to be specific to the tasks associated with volleyball (such as jumping height and overhead activities) and to the intervention ${ }^{20-22}$. The test battery was designed to measure maximal muscular strength in the lower and upper extremities and functional performance and muscular power in the lower and upper extremities and in the trunk flexors. To determine whether the tests in the test battery measured different aspects of physical performance, the relationships between the tests were investigated after the first test session. 


\section{MEASURES}

Vertical Jump. The vertical jump test was performed as a counter-movement jump, which has previously been described by Gustavsson et al. ${ }^{23}$. Players performed the jump from an upright and extended leg position with the hands placed on the waist. The players quickly bent their knees and then immediately jumped upwards for maximum height. A computerized system (MuscleLab, Ergotest Technology) using a field of infrared light (approximately $10 \mathrm{~mm}$ above the floor), serving as a contact mat, made it possible to measure the flight time. The system then converted the flight time into jump height in centimeters. The players were tested to the point at which no more improvement was made, 3-10 trials. The best attempt was used for further analysis. High test-retest reliability (ICC = 0.95 ) has been previously reported with this testing approach ${ }^{23}$.

Push-Ups. The push-up test was performed as previously described by Augustsson et al. ${ }^{24}$. The player was in a prone starting position on her toes and hands. The hands were placed shoulder-width apart with the fingers pointing forward. The elbows were held in full extension and the feet were placed $10 \mathrm{~cm}$ apart. In a continuous movement, the torso was lowered by bending the elbow joints to $90^{\circ}$ of flexion. Keeping the midsection tight and holding the head in a neutral position, the player then pressed herself back up to full elbow extension. The player used maximum speed throughout the test. Thirty-second timed pushups were documented as the number of repetitions. The test was stopped if two consecutive repetitions were unsuccessful or if the player was unable to continue. An unsuccessful repetition was regarded as one that deviated from the standard procedure. High test-retest reliability $(\mathrm{ICC}=0.93)$ has been previously reported with this testing approach ${ }^{24}$.

Sit-Ups. The sit-up test was performed as previously described by Augustsson et al. ${ }^{24}$. The player sat on the floor with the knees in $90^{\circ}$ of flexion and the feet placed $10 \mathrm{~cm}$ apart on the floor. The player's hands were clasped behind the neck and the elbows were placed against the knees. The test examiner knelt in front of the player, pushing her feet lightly against the mat. The player lowered her upper body until the scapula came in contact with the mat. The player's head was not permitted to touch the mat. The player then reversed the motion by curling back up into the starting position. The player used maximum speed throughout the test. Thirty-second timed sit-ups were documented as the number of repetitions. The test was stopped if two consecutive repetitions were unsuccessful or if the player was unable to continue. An unsuccessful repetition was regarded as one that deviated from the standard procedure. High test-retest reliability $(\mathrm{ICC}=0.93$ ) has been previously reported with this testing approach ${ }^{24}$.

As only one test leader monitored the push-up and sit-up tests, these tests were subsequently videotaped and analyzed by the test leader (first author) to ensure correct performance and number of repetitions. Minor alterations were then made (i.e., number of repetitions were reduced in final data).

1 RM Squat. The $1 \mathrm{RM}$ squat test began with a back extension warm-up exercise consisting of 20 repetitions with no extra load. Further, 20 repetitions of the squat exercise were performed with no extra load. For the test, the player stood in an upright starting position with an Olympic barbell on her shoulders placed high on the trapezius muscle. The player stood with her feet shoulder width apart, chest up and eyes fixed straight ahead. The player performed the squat by descending to a parallel position, approximately $110^{\circ}$ of knee flexion, by bending her knees and hips until the greater trochanter of the femur reached the same horizontal plane as the superior border of the patella ${ }^{16}$. The player then ascended to the starting position. The squat depth was monitored by the test leader. A board ( $2 \mathrm{~cm}$ thick) elevating the heels of the player was used, thereby facilitating a squat position. In addition, 
a tight weightlifting belt supporting the trunk was obligatory. The player was instructed to be as upright as possible. A mirror placed $1.5 \mathrm{~m}$ in front of the player enabled visual feedback. A safety squat rack was used to ensure a safe performance. The test leader stood behind the player, with his arms placed around the waist of the player during the lift to secure the exercise and to ensure that the proper form and technique was maintained. Any trials failing to meet the standardized technique criteria were discarded. The weight lifted for each trial was increased by 5-10 kg until failure occurred. $1 \mathrm{RM}$ was determined within two to seven attempts, using 1-min resting periods between trials. For the post-test, the bar was loaded to $75 \%$ of the $1 \mathrm{RM}$ performance from pre-test as the starting load. The reliability of the parallel squat test $(r=0.95)$ has previously been noted as high ${ }^{25}$.

1 RM Bench Press. The $1 \mathrm{RM}$ bench-press test began with a warm-up consisting of one set of 15 repetitions using a $10 \mathrm{~kg}$ barbell. The starting position for the bench-press test was lying supine on a bench. To standardize the bench-press technique, subjects were required to use the same hand placement for each trial, as determined by the distance between the index finger and bar striations. The bar was lowered by the individual until it touched her chest and, following a hand-clap signal from the test leader, it was then pressed into a full arm extension. The player's shoulders and buttocks had to be in contact with the bench during the test. The test leader stood over the player to secure the exercise and to ensure that the proper form and technique was maintained. Any trials failing to meet the standardized technique criteria were discarded. The weight lifted for each trial was increased by $2.5-10 \mathrm{~kg}$ until failure occurred. The $1 \mathrm{RM}$ was determined within two to six attempts, using a 1-min resting period between trials. For the post-test, the bar was loaded to $75 \%$ of the 1 RM performance from the pre-test. In a pilot study, twenty-one young female university students (mean age 25), who were not included in the study, were recruited prior to the study to perform the tests on two separate occasions within 1 week to evaluate the test-retest reliability of the $1 \mathrm{RM}$ bench-press test. The bench-press test produced a satisfactory ICC value of 0.99 with a $95 \%$ CI of (0.978-0.996).

\section{INJURY AND EXPOSURE REGISTRATION}

Volleyball training exposure was documented by coaches on a standard attendance record form. The coaches registered the total duration of each training session and game in minutes for each player during the baseline and intervention seasons. For the intervention season, the resistance-training sessions were also recorded for all teams. Completed exposure forms were returned on a monthly basis. Incomplete forms were immediately followed up by the first author. Subsequently, all forms were completed. All injuries were recorded on a standard injury form which was a modified protocol that has previously been used for injury registration in volleyball and has face validity ${ }^{4}$. The injury definition used in the present study was an injury that occurred as a result of participating in volleyball, forcing the player to leave the court for the rest of the game/training session and/or leading to an absence from or reduction in play lasting one day or more ${ }^{26}$. The severity of the injuries was classified according to Ekstrand and Gillquist ${ }^{26}$; minor injury, an injury causing absence from practice of less than one week; moderate injury, an injury causing absence from practice and games for more than a week and less than a month; major injury, an injury causing absence from practice and games for more than a month. The diagnosis determined whether the injuries were classified as either traumatic or overuse injuries. Traumatic injury was defined as an injury with a sudden onset and associated with a known trauma, whereas overuse was an injury without a known trauma and with a gradual onset; e.g., a sprain was a traumatic injury and tendinopathy was an overuse injury. The data that were collected also included whether 
the injury occurred during training or a game, the skill performed, the injured player's court position and the anatomical localization of the injury. The team coaches were responsible for the injury registration, but all injuries were followed up by a physical therapist. Subsequently, all injuries were documented but only those leading to absence were included in the injury statistics.

\section{PERIODIZED RESISTANCE TRAINING}

The training program consisted of 26 weeks of progressive resistance training divided into three phases, familiarization phase, progression phase 1 and progression phase 2 (Figure 1). All workouts started with a general warm-up of approximately $10 \mathrm{~min}$ and ended with a coolingdown period (e.g., stretching). A physical therapist (the first author), functioned as physical coach, supervised the players and was responsible for ensuring that exercise prescriptions were properly carried out and achieved during a particular workout (e.g., velocity of movement, appropriate spotting and technique, as well as safety considerations, intensity of the training and prescribed rest periods). The physical coach was also responsible for ensuring that all players attended the training session. Any missed workout was made up as quickly as possible within approximately two weeks. The players noted every training session with every exercise, every set, repetitions and training load in a training diary. The program was periodized, starting with a four-week familiarization phase with one training session per week. During this phase, the players performed exercises with approximately $70 \%$ of $1 \mathrm{RM}$ (15 repetitions) with controlled velocity. The familiarization phase consisted of a basic program which was given, as described previously, to each player in both the experimental group and the control group (Table 2). During the familiarization phase, the experimental group focused on proper technique and form and adaptation of the load. Factors such as exercise technique, equipment used, exercise order, lifting tempo, rest periods between sets and program progression were carefully explained. After the familiarization phase, the players in the experimental group were given an individual training program that was designed by the first author and included most of the exercises from the basic program (Table 3). The individual training programs were based partly on the pre-test results, but they also took account of the players' court position. The individual training programs took any previous injury into account, together with the injury screening by the visual analog scale, as mentioned in the study design section. During the subsequent 10 weeks, the aim of the training was progression (progression phase 1) with one training session per week. The training load was approximately $80 \%$ of 1 RM (10 repetitions)

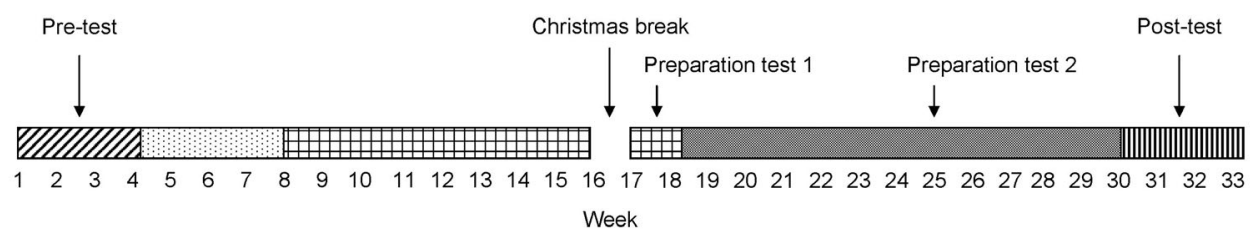

Pre-test period
Familiarization phase, approximately $70 \%$ of $1 \mathrm{RM}, 1$ training session/week
Progression phase 1 , approximately $80 \%$ of $1 \mathrm{RM}, 1$ training session/week
Progression phase 2 , approximately $90-100 \%$ of $1 \mathrm{RM}, 2$ training sessions/week
Post-test period

Figure 1. Testing and Training Schedule of the Experimental Group During the Intervention Season 
Table 3. Progression and Individualization During the Resistance Training Period for Each Player in the Experimental Group Presented by exercise, number of sets and repetitions and rest periods between sets. Phase 1 represents progression phase $1(10 \mathrm{w})$ and phase 2 represents progression phase 2 (12 w).

\begin{tabular}{|c|c|c|c|c|c|c|}
\hline \multirow[t]{2}{*}{ Exercise } & \multicolumn{2}{|c|}{ Player 1} & \multicolumn{2}{|c|}{ Player 2} & \multicolumn{2}{|c|}{ Player 3} \\
\hline & Phase 1 & Phase 2 & Phase 1 & Phase 2 & Phase 1 & Phase 2 \\
\hline Squats & $3 \times 10,2 \mathrm{~min}$ & $3 \times 1-6,3 \mathrm{~min}$ & $3 \times 10,2 \min$ & $3 \times 1-6,3 \mathrm{~min}$ & $3 \times 10,2 \min$ & $3 \times 1-6,3 \mathrm{~min}$ \\
\hline Bench press & $3 \times 10,2 \mathrm{~min} *$ & $3 \times 1-6,3 \mathrm{~min}$ & $3 \times 10,2 \mathrm{~min} *$ & $3 \times 1-6,3 \mathrm{~min}$ & $3 \times 10,2 \mathrm{~min} *$ & $3 \times 1-6,3 \mathrm{~min}$ \\
\hline Push-ups & $3 x \max , 2 \min *$ & $2 x \max 1 \min$ & $3 x \max , 2 \min *$ & $2 x \max 1 \min$ & $3 x \max , 2 \min *$ & $2 x \max 1 \min$ \\
\hline Lat pulldown & $3 \times 10,1 \mathrm{~min}$ & $2 \times 10,1 \mathrm{~min}$ & $3 \times 10,1 \mathrm{~min}$ & $2 \times 10,1 \mathrm{~min}$ & $3 \times 10,1 \mathrm{~min}$ & $2 \times 10,1 \min$ \\
\hline Seated cable row & $2 \times 10,1 \mathrm{~min}$ & & $3 \times 10,1 \mathrm{~min}$ & $2 \times 10,1 \mathrm{~min}$ & $3 \times 10,1 \mathrm{~min}$ & $2 \times 10,1 \mathrm{~min}$ \\
\hline Overhead dumbbell press & $3 \times 10^{* *}, 2 \mathrm{~min}$ & $2 \times 8,2 \min$ & $3 \times 10^{* *}, 2 \mathrm{~min}$ & $2 \times 8,2 \min$ & $3 \times 10^{* *}, 2 \mathrm{~min}$ & $2 \times 8,2 \mathrm{~min}$ \\
\hline Machine overhead press & $2 \times 10^{* *}, 1 \mathrm{~min}$ & & & & $2 \times 10^{* *}, 1 \mathrm{~min}$ & \\
\hline Cable curl (biceps) & & & $3 \times 10,30 \mathrm{sec}$ & & & \\
\hline Overhead cable extension & & & $3 \times 10,30 \mathrm{sec}$ & & & \\
\hline Bodyweight back extension & $3 \times 15^{*}, 1 \mathrm{~min}$ & $3 \times 15^{*}, 1 \mathrm{~min}$ & $3 \times 15^{*}, 1 \mathrm{~min}$ & $3 \times 15^{*}, 1 \mathrm{~min}$ & $3 \times 15^{*}, 1 \mathrm{~min}$ & $3 \times 15^{*}, 1 \mathrm{~min}$ \\
\hline Sit-ups & $3 x \max *, 1 \min$ & $3 x \max *, 1 \min$ & $3 x \max *, 1 \min$ & $3 x \max *, 1 \min$ & $3 x \max *, 1 \min$ & $3 x \max *, 1 \min$ \\
\hline \multicolumn{7}{|l|}{ Machine crunch } \\
\hline Seated leg extension & $3 \times 10,1 \mathrm{~min}$ & & $3 \times 10,1 \mathrm{~min}$ & & $3 \times 10,1 \mathrm{~min}$ & $3 \times 10,1 \mathrm{~min}$ \\
\hline Lying leg curl & & & & & $3 \times 10,1 \mathrm{~min}$ & $3 \times 10,1 \mathrm{~min}$ \\
\hline \multicolumn{7}{|l|}{ Leg press } \\
\hline One-leg leg press & $2 \times 10 /$ leg, no rest & & & & $2 \times 10 /$ leg, no rest & \\
\hline Machine seated calf raise & $3 \times 10,1 \mathrm{~min}$ & & & & & \\
\hline Bodyweight one leg squat & $2 \times 10,30 \mathrm{sec}$ & & $2 \times 10,30 \mathrm{sec}$ & & $2 \times 10,30 \mathrm{sec}$ & \\
\hline One-arm seated row & $2 \times 10 /$ arm, no rest & $2 \times 10 /$ arm, no rest & & & & \\
\hline Dumbbell row & $2 \times 10 /$ arm, no rest & & $2 \times 10 / a r m$, no rest & & & \\
\hline Bent-over lateral raise & $3 \times 10,30 \mathrm{sec}$ & $2 \times 10,30 \mathrm{sec}$ & $3 \times 10,30 \mathrm{sec}$ & $2 \times 10,30 \mathrm{sec}$ & & \\
\hline Hanging leg raise & & & $2 \times 10,30 \mathrm{sec}$ & $3 \times 15,30 \mathrm{sec}$ & & \\
\hline E ball reverse back extension & & & $2 \times 10,1 \mathrm{~min}$ & $2 \times 10,1 \mathrm{~min}$ & & \\
\hline \multicolumn{7}{|l|}{ E ball crunch } \\
\hline E ball jackknife & & & $2 \times 10,30 \mathrm{sec}$ & $2 \times 10,30 \mathrm{sec}$ & $2 \times 10,30 \mathrm{sec}$ & $2 \times 10,30 \mathrm{sec}$ \\
\hline E Ball opposite arm and leg lift & & & $2 \times 20,1 \mathrm{~min}$ & & & \\
\hline E ball prone walk out & $10,5 \mathrm{sec}$ & $10,5 \mathrm{sec}$ & & & $10,5 \mathrm{sec}$ & $10,5 \mathrm{sec}$ \\
\hline Reverse crunch & & & $2 \times 10,30 \mathrm{sec}$ & $2 \times 10,30 \mathrm{sec}$ & & \\
\hline Balance board jumping & $1 \times 10$ & $1 \times 10$ & & & & \\
\hline Barbell vertical jumps & $1 \times 10$ & $2 \times 10,2 \mathrm{~min}$ & $1 \times 10$ & $2 \times 10,2 \mathrm{~min}$ & $1 \times 10$ & $2 \times 10,2 \mathrm{~min}$ \\
\hline
\end{tabular}

* Exercises that were performed in supersets

** Exercises that were alternated every second training session.

\begin{tabular}{|c|c|c|c|c|c|c|}
\hline \multirow{2}{*}{ Exercise } & \multicolumn{2}{|c|}{ Player 4} & \multicolumn{2}{|c|}{ Player 5} & \multicolumn{2}{|c|}{ Player 6} \\
\hline & Phase 1 & Phase 2 & Phase 1 & Phase 2 & Phase 1 & Phase 2 \\
\hline Squats & $3 \times 10,2 \mathrm{~min}$ & $3 \times 1-6,3 \mathrm{~min}$ & $3 \times 10,2 \mathrm{~min}$ & $3 \times 1-6,3 \mathrm{~min}$ & $3 \times 10,2 \mathrm{~min}$ & $3 \times 1-6,3 \mathrm{~min}$ \\
\hline Bench press & $3 \times 10,2 \mathrm{~min} *$ & $3 \times 1-6,3 \mathrm{~min}$ & $3 \times 10,2 \mathrm{~min} *$ & $3 \times 1-6,3 \mathrm{~min}$ & $3 \times 10,2 \mathrm{~min} *$ & $3 \times 1-6,3 \mathrm{~min}$ \\
\hline Push-ups & $3 x \max , 2 \min *$ & $2 x \max 1 \min$ & $3 x \max , 2 \min *$ & $2 x \max 1 \min$ & $3 x \max , 2 \min *$ & $2 x \max 1 \min$ \\
\hline Lat pulldown & $3 \times 10,1 \mathrm{~min}$ & $2 \times 10,1 \mathrm{~min}$ & $3 \times 10,1 \mathrm{~min}$ & $2 \times 10,1 \mathrm{~min}$ & $3 \times 10,1 \mathrm{~min}$ & $2 \times 10,1 \mathrm{~min}$ \\
\hline Seated cable row & $3 \times 10,1 \mathrm{~min}$ & $2 \times 10,1 \mathrm{~min}$ & $2 \times 10,1 \mathrm{~min}$ & & $2 \times 10,1 \mathrm{~min}$ & \\
\hline Overhead dumbbell press & $3 \times 10^{* *}, 2 \mathrm{~min}$ & $2 \times 8,2 \min$ & $3 \times 10^{* *}, 2 \mathrm{~min}$ & $2 \times 8,2 \min$ & $3 \times 10,2 \mathrm{~min}$ & $2 \times 8,2 \min$ \\
\hline Machine overhead press & $2 \times 10^{* *}, 1 \mathrm{~min}$ & & $2 \times 10^{* *}, 1 \mathrm{~min}$ & & & \\
\hline \multicolumn{7}{|l|}{ Cable curl (biceps) } \\
\hline \multicolumn{7}{|l|}{ Overhead cable extension } \\
\hline Bodyweight back extension & $3 \times 15^{*}, 1 \mathrm{~min}$ & $3 \times 15^{*}, 1 \mathrm{~min}$ & $3 \times 15 *, 1 \mathrm{~min}$ & $3 \times 15^{*}$ & $3 \times 15^{*}, 1 \mathrm{~min}$ & $3 \times 15^{*}, 1 \mathrm{~min}$ \\
\hline Sit-ups & $3 x \max ^{*}, 1 \min$ & $3 x \max *, 1 \min$ & $3 x_{\max }^{*}, 1 \min$ & $3 x \max ^{*}, 1 \min$ & $3 x \max ^{*}, 1 \min$ & $3 x \max *, 1 \min$ \\
\hline Machine crunch & $3 \times 15$ & $2 \times 15$ & & & & \\
\hline Seated leg extension & & & $3 \times 10,1 \mathrm{~min}$ & & & \\
\hline Lying leg curl & & & $3 \times 10,1 \mathrm{~min}$ & $3 \times 10,1 \mathrm{~min}$ & & \\
\hline Leg press & $3 \times 10,1 \mathrm{~min}$ & $2 \times 10,1 \mathrm{~min}$ & & & $3 \times 10,1 \mathrm{~min}$ & $2 \times 10,1 \mathrm{~min}$ \\
\hline One-leg leg press & & & $2 \times 10 /$ leg, no rest & & & \\
\hline Machine seated calf raise & & & $3 \times 10,1 \mathrm{~min}$ & & & \\
\hline Bodyweight one leg squat & & & $2 \times 10,30 \mathrm{sec}$ & & $2 \times 10,30 \mathrm{sec}$ & \\
\hline \multicolumn{7}{|l|}{ One-arm seated row } \\
\hline \multicolumn{7}{|l|}{ Dumbbell row } \\
\hline Bent-over lateral raise & $3 \times 10,30 \mathrm{sec}$ & $2 \times 10,30 \mathrm{sec}$ & & & $3 \times 10,30 \mathrm{sec}$ & $2 \times 10,30 \mathrm{sec}$ \\
\hline Hanging leg raise & $2 \times 10,30 \mathrm{sec}$ & $3 \times 15,30 \mathrm{sec}$ & & & $2 \times 10,30 \mathrm{sec}$ & $3 \times 15,30 \mathrm{sec}$ \\
\hline E ball reverse back extension & & & $2 \times 10,1 \mathrm{~min}$ & $2 \times 10,1 \mathrm{~min}$ & & \\
\hline E ball crunch & & & & & & \\
\hline E ball jackknife & & & $2 \times 10,30 \mathrm{sec}$ & $2 \times 10,30 \mathrm{sec}$ & & \\
\hline E Ball opposite arm and leg lift & & & & & & \\
\hline $\mathrm{E}$ ball prone walk out & & & $10,5 \mathrm{sec}$ & $10,5 \mathrm{sec}$ & & \\
\hline Reverse crunch & & & & & & \\
\hline Balance board jumping & & & $1 \times 10$ & $1 \times 10$ & & \\
\hline Barbell vertical jumps & $1 \times 10$ & $2 \times 10,2 \mathrm{~min}$ & $1 \times 10$ & $2 \times 10,2 \mathrm{~min}$ & $1 \times 10$ & $2 \times 10,2 \mathrm{~min}$ \\
\hline
\end{tabular}

* Exercises that were performed in supersets.

** Exercises that were alternated every second training session 
Table 3. continued

\begin{tabular}{|c|c|c|c|c|c|c|c|c|}
\hline \multirow[t]{2}{*}{ Exercise } & \multicolumn{2}{|c|}{ Player 7} & \multicolumn{2}{|c|}{ Player 8} & \multicolumn{2}{|c|}{ Player 9} & \multicolumn{2}{|c|}{ Player 10} \\
\hline & Phase 1 & Phase 2 & Phase 1 & Phase 2 & Phase 1 & Phase 2 & Phase 1 & Phase 2 \\
\hline Squats & $3 \times 10,2 \mathrm{~min}$ & $3 \times 1-6,3 \mathrm{~min}$ & $3 \times 10,2 \mathrm{~min}$ & $3 \times 1-6,3 \mathrm{~min}$ & $3 \times 10,2 \mathrm{~min}$ & $3 \times 1-6,3 \mathrm{~min}$ & $3 \times 10,2 \mathrm{~min}$ & $3 \times 1-6,3 \mathrm{~min}$ \\
\hline Bench press & $3 \times 10,2 \min ^{*}$ & $3 \times 1-6,3 \min$ & $3 \times 10,2$ min* & $3 \times 1-6,3 \mathrm{~min}$ & $3 \times 10,2 \min ^{*}$ & $3 \times 1-6,3 \mathrm{~min}$ & $3 \times 10,2 \min ^{*}$ & $3 \times 1-6,3 \min$ \\
\hline Push-ups & $3 x \max , 2 \min *$ & $2 \times \max 1 \min$ & $3 x \max , 2 \min *$ & $2 x \max 1 \min$ & $3 x \max , 2 \min ^{*}$ & $2 x \max 1 \min$ & $3 x \max , 2$ min* & $2 x \max 1 \min$ \\
\hline Lat pulldown & $3 \times 10,1 \mathrm{~min}$ & $2 \times 10,1 \mathrm{~min}$ & $3 \times 10,1 \mathrm{~min}$ & $2 \times 10,1 \mathrm{~min}$ & $3 \times 10,1 \mathrm{~min}$ & $2 \times 10,1 \mathrm{~min}$ & $3 \times 10,1 \mathrm{~min}$ & $2 \times 10,1 \mathrm{~min}$ \\
\hline Seated cable row & $3 \times 10,1 \mathrm{~min}$ & $2 \times 10,1 \mathrm{~min}$ & $3 \times 10,1 \mathrm{~min}$ & $2 \times 10,1 \mathrm{~min}$ & $3 \times 10,1 \mathrm{~min}$ & $2 \times 10,1 \mathrm{~min}$ & $3 \times 10,1 \mathrm{~min}$ & $2 \times 10,1 \mathrm{~min}$ \\
\hline Overhead dumbbell press & $3 \times 10,2 \mathrm{~min}$ & $2 \times 8,2 \min$ & & & $3 \times 10,2 \mathrm{~min}$ & $2 \times 8,2 \min$ & $3 \times 10^{* *}, 2 \mathrm{~min}$ & $2 \times 8,2 \min$ \\
\hline Machine overhead press & & & $2 \times 10,1 \mathrm{~min}$ & $3 \times 10,1 \mathrm{~min}$ & & & $2 \times 10^{* *}, 1 \mathrm{~min}$ & \\
\hline Cable curl (biceps) & & & & & $3 \times 10,30 \mathrm{sec}$ & & & \\
\hline Overhead cable extension & & & & & $3 \times 10,30 \mathrm{sec}$ & & & \\
\hline Bodyweight back extension & $3 \times 15^{*}, 1 \mathrm{~min}$ & $3 \times 15^{*}, 1 \mathrm{~min}$ & $3 \times 15^{*}, 1 \mathrm{~min}$ & $3 \times 15^{*}, 1 \mathrm{~min}$ & $3 \times 15^{*}, 1 \mathrm{~min}$ & $3 \times 15^{*}, 1 \mathrm{~min}$ & $3 \times 15^{*}, 1 \mathrm{~min}$ & $3 \times 15^{*}, 1 \mathrm{~min}$ \\
\hline $\begin{array}{l}\text { Sit-ups } \\
\text { Machine crunch }\end{array}$ & $\begin{array}{l}3 \mathrm{xmax} *, 1 \mathrm{~min} \\
3 \times 15\end{array}$ & $3 x \max *, 1$ min & $3 x \max *, 1$ min & $3 x \max *, 1 \min$ & $3 \mathrm{xmax}^{*}, 1 \mathrm{~min}$ & $3 x \max *, 1 \min$ & $3 x \max ^{*}, 1 \mathrm{~min}$ & $3 x \max *, 1 \min$ \\
\hline Seated leg extension & & & & & $3 \times 10,1 \mathrm{~min}$ & $3 \times 10,1 \mathrm{~min}$ & $3 \times 10,1 \mathrm{~min}$ & \\
\hline Lying leg curl & & & & & & & $3 \times 10,1 \mathrm{~min}$ & $3 \times 10,1 \mathrm{~min}$ \\
\hline Leg press & $3 \times 10,1 \mathrm{~min}$ & $2 \times 10,1 \mathrm{~min}$ & $3 \times 10,1 \mathrm{~min}$ & $2 \times 10,1 \mathrm{~min}$ & & & & \\
\hline One-leg leg press & & & $2 \times 10 /$ leg, no rest & & & & $2 \times 10 /$ leg, no rest & \\
\hline Machine seated calf raise & & & & & & & $3 \times 10,1 \mathrm{~min}$ & \\
\hline Bodyweight one leg squat & $2 \times 10,30 \mathrm{sec}$ & & $2 \times 10,30 \mathrm{sec}$ & & & & $2 \times 10,30 \mathrm{sec}$ & \\
\hline One-arm seated row & & & & & & & & \\
\hline Dumbbell row & & & & & $2 \times 10 / a r m$, no rest & $2 \times 10 /$ arm, no rest & & \\
\hline Bent-over lateral raise & $3 \times 10,30 \mathrm{sec}$ & $2 \times 10,30 \mathrm{sec}$ & & & & & & \\
\hline Hanging leg raise & & & & & $2 \times 10,30 \mathrm{sec}$ & $3 \times 15,30 \mathrm{sec}$ & & \\
\hline E ball reverse back extension & & & $2 \times 10,1 \mathrm{~min}$ & $2 \times 10,1 \mathrm{~min}$ & & & & \\
\hline E ball crunch & $2 \times 10$ & $3 \times 10$ & & & & & & \\
\hline E ball jackknife & & & & & & & & \\
\hline E Ball opposite arm and leg lift & & & & & & & & \\
\hline E ball prone walk out & & & $10,5 \mathrm{sec}$ & $10,5 \mathrm{sec}$ & & & $10,5 \mathrm{sec}$ & $10,5 \mathrm{sec}$ \\
\hline Reverse crunch & & & & & $2 \times 10,30 \mathrm{sec}$ & $2 \times 10,30 \mathrm{sec}$ & & \\
\hline Balance board jumping & & & & & & & & \\
\hline Barbell vertical jumps & $1 \times 10$ & $2 \times 10,2 \mathrm{~min}$ & $1 \times 10$ & $2 \times 10,2 \mathrm{~min}$ & $1 \times 10$ & $2 \times 10,2 \mathrm{~min}$ & & \\
\hline
\end{tabular}

* Exercises that were performed in supersets.

** Exercises that were alternated every second training session.

and was increased, if possible, every two weeks to maintain the $80 \%$ level in order to gain strength. After progression phase 1, progression phase 2 was initialized and consisted of 12 weeks of high-intensity resistance training, with two training sessions per week, focusing on maximum strength and performance. The individual program did not change during this phase, but the training intensity increased. The players increased the training load to $90-100 \%$ of $1 \mathrm{RM}$ in some of the multiple-joint exercises, such as the squat and the bench press. The players were instructed to complete all repetitions in all sets, even if assistance was required for several repetitions (so-called forced repetition). All training sessions were recorded (i.e., exercises, training load, number of sets and repetitions and total duration of training) in the athlete's training diary. The players performed all bench-press and squat training in a free weight using a standard Olympic 20-kg barbell. The players in the experimental group were tested by the physical coach, in week 12 and in week 20, in squat, bench press, push-ups and sit-ups to determine any flaws and to evaluate performance enhancement.

Initial training loads $(\mathrm{kg})$ were based on the pre-test results and also on the training performed during the familiarization phase of the study, using approximate percentages of the 1 RM. Then, using standard trial and error methods (i.e., unload if too few repetitions were performed and load if too many repetitions were performed), the loads for each set were modulated based on performance with reference to the target $1 \mathrm{RM}$. The physical coach was responsible for this careful modulation of the training load progression. The progress by each athlete, in terms of training intensity, volume and type of exercises, was supervised and outlined individually by the physical coach. Most of the exercises were performed simultaneously by pairs of players. The players were instructed to give verbal encouragement to each other and to secure the exercises. Some exercises were performed using alternating sets (supersets) of two exercises (e.g., bench press and push-ups) to increase the time efficiency of the workout by decreasing the amount of rest between sets. These alternating 
sets were performed until progression phase 2 started.

\section{STATISTICAL METHODS}

To evaluate changes in physical performance, sample size calculations and the power to detect a difference of $50 \%$, at the $95 \%$ significance level, between the experimental group and the control group were determined by power analysis. A 50\% mean difference in the test battery (vertical jump, 1 RM squat, 1 RM bench press, push-ups and sit-ups) was used for the power analysis. The reference values against which these differences were calculated came from previous training studies by Kraemer et al. ${ }^{27}$ (vertical jump and bench press), Augustsson et al. ${ }^{28}$ (squat), and Bell et al. ${ }^{29}$ (push-ups and sit-ups).The number of players required to obtain sufficient power $(90 \%)$ was achieved before the start of the study ( $\mathrm{n}=17$ /group).

The descriptive data relating to the players are based on registration at the pre-test session. Pre- and post-intervention data were analyzed with Wilcoxon's signed-rank test. The MannWhitney $U$ test was used to determine significant differences between the groups for the various tests in the test battery. Standardized Z-scores were used when calculating the total score for the test battery and the total score differences were analyzed using the One-Way ANOVA test. The relationships between the tests in the test battery were also investigated, using Pearson's correlation coefficient, to determine whether they measured different aspects of physical performance. Injury incidence rates were calculated, together with a $95 \%$ confidence interval (CI), taking exposure into account and expressed as the number of injury occurrences per 1,000 h of volleyball practice and game. Injury prevalence was calculated as the number of injury occurrences per volleyball player. Fisher's exact test was used to compare the percentage of injured players in the experimental group and the control group in the baseline season and the intervention season. The SPSS statistical software package, version 15 (SPSS Inc., Chicago, U.S.A), was used for statistical calculations. The level of significance was set at $\mathrm{p}<0.05$.

\section{RESULTS}

Twenty-seven players (10 in the experimental group and 17 in the control group) completed the study. Three players dropped out of the experimental group, two due to quitting the volleyball team and one due to other sports participation conflicting with the resistancetraining sessions. Three players dropped out of the control group for unknown reasons. No differences were found between the groups in terms of age, height or weight. The body weight noted at the post-test did not differ from that at pre-test registration in either of the groups. Three of the players in the control group were unable to attend the post-test occasion due to injuries. These players were still included in the injury data registration and thus were not excluded from the study.

\section{PHYSICAL PERFORMANCE}

No significant differences were noted at the pre-test for any individual tests or for the total score $(\mathrm{p}=0.495)$ for the test battery between the experimental group and control group. At post-test, the players in the experimental group had improved significantly more than the players in the control group in the squat $(\mathrm{p}<0.001)$, bench press $(\mathrm{p}=0.042)$, push-ups $(\mathrm{p}=0.002)$ and sit-ups $(\mathrm{p}<0.001)$ but not in the vertical jump test $(\mathrm{p}=0.412)($ Table 4$)$. The total score for the test battery was also significantly higher in the experimental group compared with the control group ( $\mathrm{p}<0.001$ ). Table 4 shows the pre and post scores for the individual tests in the test battery. 
Table 4. Mean ( \pm SD) Results of the Vertical Jump, 1 Repetition Maximum Squat, 1 Repetition Maximum Bench Press, 30-sec Timed Push-Ups and 30-sec Timed Sit-ups Tests

\begin{tabular}{lclllll} 
& \multicolumn{7}{l}{ Experimental $(\mathbf{n}=\mathbf{1 0})$} & \multicolumn{2}{l}{ Control $(\mathbf{n}=\mathbf{1 4})$} \\
\hline & Pre & Post & Change $(\boldsymbol{\%})$ & Pre & Post & Change (\%) \\
\hline Vertical jump $(\mathrm{cm})$ & $26( \pm 4)$ & $28( \pm 4) \ddagger$ & 8 & $27( \pm 4)$ & $28( \pm 4)$ & 4 \\
\hline Squat $(\mathrm{kg})$ & $42( \pm 14)$ & $70( \pm 13)^{*} \ddagger$ & 67 & $50( \pm 14)$ & $56( \pm 17)$ & 12 \\
\hline Bench press $(\mathrm{kg})$ & $31( \pm 5)$ & $36( \pm 6)^{*} \ddagger$ & 16 & $32( \pm 5)$ & $34( \pm 6) \ddagger$ & 6 \\
\hline Push-ups & $9( \pm 4)$ & $20( \pm 5)^{*} \ddagger$ & 122 & $10( \pm 4)$ & $13( \pm 5)$ & 30 \\
\hline Sit-ups & $11( \pm 3)$ & $17( \pm 3)^{*} \ddagger$ & 55 & $13( \pm 3)$ & $14( \pm 2)$ & 8 \\
\hline
\end{tabular}

*Difference in increase between groups $(\mathrm{p}<0.05)$.

†Intragroup difference $(\mathrm{p}<0.05)$.

\section{PRE- AND POST-CORRELATION BETWEEN TESTS}

A significant relationship was found between the vertical jump test and the squat test $(r=$ $0.42, p=0.026)$ at the pre-test. No significant correlations were found for any of the other tests in the test battery at the pre-test.

\section{COMPLIANCE}

Thirty-five percent (6/17) of the players from the control group performed the resistancetraining program to which they were assigned during the intervention season, with $50 \%$ or more compliance (i.e., the total number of resistance training sessions) during the season. All the players (excluding drop-outs, $\mathrm{n}=10$ ) from the experimental group carried out the resistance-training program during the entire season. Eighty percent of these players had at least 50\% compliance. When comparing players who were compliant, we found that the experimental group improved its physical performance for the total test battery significantly more than the control group $(\mathrm{p}=0.002)$.

INJURY DATA

\section{Baseline Season 2006-2007}

During 3,546 hours of exposure time (practice and games), nine (29\%) players sustained 15 injuries during the baseline season. The injury incidence for the experimental group was 5.3 injuries with a 95\% CI of -1.2-12.2. The injury incidence for the control group was 3.8 injuries with a 95\% CI of 1.2-6.4. The injury incidence and prevalence are shown in Table 5. There was no significant difference in the proportion of injured players between the experimental group and control group $(\mathrm{p}=0.437)$. The most common injury was localized in the knee area (seven of 15 injuries). There were also injuries to the ankles $(2 / 15)$, shoulders $(2 / 15)$, lower leg (2/15) and wrist (2/15). Of all the injuries, $71 \%$ were overuse symptoms and $29 \%$ were of a traumatic nature. Of the four traumatic injuries, one (1/4) occurred during contact with another player. $72 \%$ of the injuries were of moderate severity, while $21 \%$ were of minor severity and $7 \%$ were of major severity.

\section{Intervention Season 2007-2008}

For the intervention season, an exposure time of 3,242 hours was noted during which six players sustained eight injuries. The injury incidence for the experimental group was zero injuries. The injury incidence for the control group was 3.7 injuries with a 95\% CI of 0.8 - 
6.6. The injury incidence and prevalence are shown in Table 5. There was a significant difference $(\mathrm{p}=0.042)$ in the proportion of injured players between the experimental group and the control group in the intervention season. Fifty percent (4/8) were ankle injuries of a traumatic nature, whereas the other injuries were overuse injuries located in the knee $(2 / 8)$, shoulder (1/8) and thigh (1/8) areas. Fifty percent (2/4) of the traumatic injuries occurred during contact with another player. Seventy-five percent of the injuries were evenly distributed between minor and moderate severity, while $25 \%$ were of major severity.

Table 5. Injury Incidence (Number of Injuries per 1000 hours of Exposure) and Prevalence with $95 \% \mathrm{Cl}$, Frequency and Rate of Injured Players in the Control $(n=17)$ and the Experimental Group $(n=10)$ During the Baseline and Intervention Season

\begin{tabular}{lllllll}
\hline & \multicolumn{5}{l}{ Baseline season } & \multicolumn{2}{l}{ Intervention season } \\
\hline & Control & Experimental & Total & Control & Experimental & Total \\
\hline Injury incidence $(95 \% \mathrm{CI})$ & $3.8(1.2-6.4)$ & $5.3(-1.2-12.2)$ & 4.3 & $3.7(0.8-6.6)$ & $0(0)$ & 2.5 \\
\hline Injury prevalence $(95 \% \mathrm{CI})$ & $0.6(0.2-1.0)$ & $0.5(-0.1-1.1)$ & 0.6 & $0.5(0.1-0.8)$ & $0(0)$ & 0.3 \\
\hline Injury frequency & 10 & 5 & 15 & 8 & 0 & 8 \\
\hline Injured players & 7 & 3 & 10 & 6 & 0 & 6 \\
\hline
\end{tabular}

\section{DISCUSSION}

The main finding in this study was the significant improvement in strength and power in the experimental group, with individual and supervised resistance training, in comparison with the control group performing standard group training that did not address individual needs and/or were only indirectly supervised. The present study revealed no significant differences at the pre-test for any tests between the experimental group and the control group. At posttest, the players in the experimental group had improved significantly more than the players in the control group in all tests except the vertical jump test. However, the improvements noted in the vertical jump test between pre- and post-test were significant in the experimental group but not in the control group. The pre-test sessions were performed during the preseason and after the summer break. Consequently, the players' jumping performance was probably poorer due to training reductions during the summer and then, after one season of regular training, the players in both groups experienced normal adaptation to sport. Previous studies have shown that the physical performance, such as jumping performance and throwing velocity, were enhanced during one season of ordinary training or preparation for competition in sports ${ }^{21,30}$ and that interruptions in normal training can result in physical capacity reductions $^{31}$. Since jumping is one of the most important tasks in volleyball, it is likely that this ability will improve during one season of volleyball training. The lack of significant differences between groups when it came to jumping performance in the present study could therefore possibly be explained by this fact.

Previously, few studies have examined the effect of entire in-season resistance training for physical performance and injury prevention in sport. Designing an optimal resistancetraining program for sport can be a complex process in itself. The resistance training prescribed for the players at the beginning (i.e., basic program) of the present study was the same for both groups. A simple program design is recommended when resistance training is introduced $^{12}$. Consequently, the basic program in the present study consisted of no more than nine exercises and focused on the correct technique and form and adaptation of the load. After the familiarization phase, the players in the experimental group received an individual 
training program. The program included most of the exercises from the basic program with additional and individual exercises. Improvements in physical performance and associated physiological adaptations are correlated to the intensity and number of repetitions performed $^{32}$. It has been recommended that training with loads corresponding to $80-85 \%$ of $1 \mathrm{RM}$ or more are effective for increasing maximum strength ${ }^{32}$. Although strength also increases using loads corresponding to $70-80 \%$ of $1 \mathrm{RM}$, it is believed that this range may not be as effective in increasing maximum strength in advanced strength-trained athletes compared with heavier loading (e.g., $85 \%$ of $1 \mathrm{RM}$ ). Since the athletes in the present study were inexperienced in resistance training, we therefore set the starting load at $70 \%$ of $1 \mathrm{RM}$, in order to begin gradually, learn the proper technique and allow sufficient recovery time. However, the more advanced the athletes become in performing the exercises, the more variation (i.e., specific training cycles) may be necessary to avoid performance plateaus ${ }^{12}$. For this reason, the training load was increased to approximately $80 \%$ of $1 \mathrm{RM}$ (progression phase 1) after the familiarization phase to augment progression. The training load was then increased, if possible, every two weeks to maintain the $80 \%$ level and optimize strength gains. Moreover, after progression phase 1, the load increased even more to an intensity corresponding to $90-100 \%$ of $1 \mathrm{RM}$ (progression phase 2) in some of the multiple-joint exercises. Progression phase 2 consisted of 12 weeks of heavy resistance training, with two training sessions a week, focusing on maximum strength and performance mainly in the multiple-joint exercises. Multiple-joint exercises require rapid force production and have been widely used for strength and power training ${ }^{20,33}$. Because vertical jump capacity (a multiple-joint movement) is critical for success in volleyball, multiple-joint exercises were included in the present study, as well as ordinary explosive strength exercises, such as the barbell jump squat. However, the multiple-joint exercises require additional time to learn and the proper technique compared with single-joint exercises. To secure safety, it is recommended that multiple-joint exercises are performed early in the workout session when fatigue is minimal ${ }^{12}$. For this reason, the multiple-joint exercises included in the present study training regimens were placed first in each training session.

The players in the present study were instructed to complete all the repetitions in all the sets, even if assistance was required with several repetitions. This training method is known as forced repetitions. Previous studies have suggested that, at least for experienced strength athletes, the forced-repetition and the repetition-to-failure method may be superior to the more traditional method when it comes to optimizing hormonal response and producing greater strength gains ${ }^{34-36}$. In the present study, the players performed exercises to failure and the forced-repetition method in some of the exercises in progression phases 1 and 2 with the aim of optimizing strength gains and avoiding stagnation. It has also been noted that the systematic use of various loading strategies is needed for optimal strength and local muscular endurance ${ }^{37}$ and a periodized training protocol is recommended ${ }^{27}$. The training programs in the present study were therefore periodized in terms of frequency, duration, intensity, volume and rest intervals. Exercise testing was carried out by the physical coach twice during the study period to determine strength deficits and evaluate the athletes' performance enhancement and their progress with the programs.

It has been suggested that tests using free weights reflect the functional strength in sports $^{20,33}$. For example, maximum squat strength has been shown to have a strong relationship with vertical jump height ${ }^{20}$. The test battery used in the present study was constructed to reflect the exercises performed by the players in order to fully to detect eventual improvements as a result of training. The test battery was also thought to be sport specific and related to the specific injury panorama in volleyball. Since only one low 
significant relationship $(\mathrm{r}=0.42)$ was found (the vertical jump test and the squat test) between individual tests, the test battery appeared to measure different aspects of physical performance.

Resistance-training programs that are individualized to meet specific training goals are thought to be superior to non-individual programs ${ }^{12}$. Individual programs should depend on factors such as initial performance and health status ${ }^{38}$. Many issues need to be taken into account when designing resistance-training programs for athletes as well as non-athletes. For example, the injury profile, the athletes' individual strengths/weaknesses, the demands of the sport, court position and physical fitness (e.g., weight, height) all require attention when designing resistance-training programs ${ }^{12}$. The training programs in the present study were individualized on the basis of the pre-test results and also while taking the players' court position, previous injuries and the injury screening into account. All training performed by the players was recorded in both groups. However, the intensity of the resistance training was not recorded in the control group. All the players in the experimental group documented their exercises, training volume and training intensity in a diary. The fact that the players in the experimental group recorded each training session more thoroughly may have influenced the higher rate of compliance with the program documented in the experimental group compared with the control group. The use of a training diary could have helped the athlete to remind herself of the current exercise prescription, training load and number of sets and repetitions. The diary could also have promoted goal-oriented issues. Using the visible achievement of a diary (e.g., becoming aware of progress made) might also have facilitated and encouraged the athlete to complete every training session to the fullest.

The present study provides more evidence supporting the importance of supervision during resistance training in order to optimize strength gains. According to the National Strength and Conditioning Association (NSCA) ${ }^{39}$, strength and conditioning programs must provide adequate appropriate supervision with well-qualified, trained personnel. One previous study has examined the effect of direct supervision by a trained strength and conditioning coach on measures of muscular strength and power in young rugby league players ${ }^{19}$. The players were directly coach-to-athlete supervised and compared with players supervised by a team manager who was not trained in strength and conditioning coaching. The outcome showed greater training adherence and increased strength gains for the players in the direct coach-to-athlete supervised group, which underlines the importance of having educated strength and conditioning coaches in sports. However, the opportunity for athletes to have direct coach-to-athlete supervision in strength and conditioning programs, as well as the sport-specific training routine, is limited, especially in team sports. The challenge for the coach when strength and conditioning programs are being introduced is therefore to have quick access to those in the greatest need of support.

We found greater compliance with the resistance-training sessions in the experimental group compared with the control group. We do not know whether more players in the control group would have benefited from the program if they had taken part in more training sessions. However, the compliant players in the experimental group improved their physical performance significantly more than the compliant players in the control group. In spite of this, due to the restricted sample size in the compliant control group, it is not possible to determine whether this difference was due to the individualized and supervised program or was just a random effect. Nevertheless, we believe that the higher compliance rate seen in the experimental group might be due to the direct supervision of each training session. Further, we believe that, if a training instructor stands waiting for the players, it is probably more motivating to attend the sessions. Taken together, we believe that compliance with 
prevention programs plays a very important role when it comes to the effectiveness of a program and that supervision and individualization can enhance the players' compliance.

Statistical power was calculated on an appropriate sample size to detect differences in physical performance between groups, but the resistance-training program was also designed to prevent injuries. The total injury incidence $(4.3$ injuries $/ 1,000 \mathrm{~h})$ documented during the baseline season in the present study is higher than has previously been reported for volleyball ${ }^{40,41}$. For example, Verhagen et al. ${ }^{41}$ reported an overall injury incidence of 2.6 injuries/1,000 hours for volleyball players. However, their study comprised 486 players in both the second and third Dutch national volleyball divisions, which is a much larger sample size than in the present study. There are no studies that have registered the injury incidence in the third division of volleyball alone, which makes comparisons with other studies difficult. There was a high frequency of overuse injuries (71\% noted during the baseline season) in the present study. This may be due to the fact that volleyball players in the third division are not exposed to game play, where traumatic injuries mostly occur, to the same extent as players in higher divisions ${ }^{40,41}$. In addition, only one of the four traumatic injuries occurred in contact with another player during the baseline season. The contact traumatic injuries in volleyball, such as ankle sprains, are often the results of incorrect take-off and landing techniques ${ }^{40}$ and the incidence of both contact and non-contact traumatic injuries could therefore be reduced by technical training ${ }^{7}$. It has yet to be established whether resistance training reduces the incidence of these injuries. The majority of the injuries were localized in the knee, ankle and shoulder area, which appears to be in accordance with the injury location generally seen in volleyball ${ }^{4,41,42}$. No significant difference was found in the proportion of injured players after the baseline season, whereas there was a significant difference in the proportion of injured players during the intervention season between the experimental group and the control group. When it comes to the injury reduction in the experimental group, we speculate that the cause could comprise of several factors. For some players, improved performance (e.g., strength increases) may have prevented injury. Moreover, improved technique (e.g., improved neuromuscular performance) may have reduced injurious forces for other players. Further, the level of awareness may have increased among players when it comes to injury prevention. This may have contributed to a change in attitude towards preventive action. However, the number of injuries is limited and the effect of the program on the injury panorama cannot be assessed reliably in the present study.

\section{METHODOLOGICAL ISSUES}

With respect to the study design, players in the present study were not randomized. However, it is difficult to split the team members into different training regimens without the possibility of interference. Consequently, we chose to randomize the teams instead in order to reduce the risk of confounding factors. One potential weakness of the present study is the relatively small sample size. Power for detecting injury incidence, prevalence and frequency was limited and we are unable to draw any definite conclusions from the injury results. There were six drop-outs (15\%), which has to be regarded as an acceptable number when considering the length of the study (two seasons and 26 weeks of intervention). Moreover, the main strength of the study is the players' compliance with the study and the intervention protocol. Previous studies have indicated that compliance with a training program is an important factor and a real effort must be made to motivate participants to carry out the program $^{14,15}$. For example, one study with a large sample size, comprising 2,020 football players, was not able to detect any effect using an injury-prevention program ${ }^{15}$. The lack of effect was thought to be a result of poor compliance. A smaller sample size might contribute 
to higher quality and greater compliance with the program and may thus be more effective in identifying differences. Taken together, although a large sample size is preferable statistically and methodologically, there is probably a risk that the intervention (when it comes to information, supervision, placebo effect, and so on) will be diluted and more difficult to manage compared with a small sample study. As mentioned earlier in the Discussion section, the players in the experimental group had improved significantly more than the players in the control group in all tests except the vertical jump test. Whether statistically significant differences have any clinical relevance is always a matter of opinion $^{43}$. In our study, however, the pre/post differences for the squat, bench press, pushups and sit-ups tests were $67 \%, 16 \%, 122 \%$ and $55 \%$, respectively, in the experimental group which we believe represent clinically meaningful improvements.

\section{CONCLUSION}

The present study shows that female volleyball players can improve strength and power during the competition season by implementing a resistance training program. Both individualization and supervision of resistance training seem to improve greater training adherence and strength gains compared with non-individualised and unsupervised training. Also, supervision alone could improve compliance and thus be an explanation for the differences seen in the present study.

\section{ACKNOWLEDGEMENT}

This study was supported by a grant from the Swedish National Centre for Research in Sports and grants from The Lundgrenska Foundations and The Royal Society of Arts and Science in Göteborg.

\section{REFERENCES}

1. Arendt, E. and Dick, R., Knee Injury Patterns among Men and Women in Collegiate Basketball and Soccer: NCAA Data and Review of Literature, The American Journal of Sports Medicine, 1995, 23, 694-701.

2. Myklebust, G., Maehlum, S., Holm, I. and Bahr, R., A Prospective Cohort Study of Anterior Cruciate Ligament Injuries in Elite Norwegian Team Handball, Scandinavian Journal of Medicine and Science in Sports, 1998, 8, 149-153.

3. Barber-Westin, S.D., Noyes, F.R. and Galloway, M., Jump-Land Characteristics and Muscle Strength Development in Young Athletes: A Gender Comparison of 1140 Athletes 9 to 17 Years of Age, The American Journal of Sports Medicine, 2006, 34, 375-384.

4. Augustsson, S.R., Augustsson, J., Thomeé, R. and Svantesson U., Injuries and Preventive Actions in Elite Swedish Volleyball, Scandinavian Journal of Medicine and Science in Sports, 2006, 16, 433-440.

5. Árnason, A., Andersen, T.E., Holme, L., Engebretsen, R. and Bahr R., Prevention of Hamstring Strains in Elite Soccer: An Intervention Study, Scandinavian Journal of Medicine and Science in Sports, 2003, 18, 4048.

6. Askling, C., Karlsson J. and Thorstensson, A., Hamstring Injury Occurrence in Elite Soccer Players after Preseason Resistance Training with Eccentric Overload, Scandinavian Journal of Medicine and Science in Sports, 2003, 13, 244-250.

7. Reeser, J.C., Verhagen, E., Briner, W.W., Askeland, T.I. and Bahr, R., Strategies for the Prevention of Volleyball Related Injuries, British Journal of Sports Medicine, 2006, 40, 594-600.

8. Frohm, A., Saartok, T., Halvorsen, K. and Renström, P., Eccentric Treatment for Patellar Tendinopathy: A Prospective Randomized Short-Term Pilot Study of Two Rehabilitation Protocols, British Journal of Sports Medicine, 2007; 41:e7. 
9. Niederbracht, Y., Shim, A.L., Sloniger, M.A., Paternostro-Bayles, M. and Short, T.H., Effects of a Shoulder Injury Prevention Strength Training Program on Eccentric External Rotator Muscle Strength and Glenohumeral Joint Imbalance in Female Overhead Activity Athletes, Journal of Strength and Conditioning Research, 2008, 22, 140-145.

10. Stickley, C.D., Hetzler, R.K., Freemyer, B.G. and Kimura, I.F., Isokinetic Peak Torque Ratios and Shoulder Injury History in Adolescent Female Volleyball Athletes, Journal of Athletic Training, 2008, 43, 571-577.

11. Eitzen, I., Holm, I. and Risberg, MA., Preoperative Quadriceps Strength Is a Significant Predictor of Knee Function Two Years after Anterior Cruciate Ligament Reconstruction, British Journal of Sports Medicine, 2009, 43, 371-376.

12. Kraemer, W.J. and Ratamess, N.A., Fundamentals of Resistance Training: Progression and Exercise Prescription, Medicine and Science in Sports and Exercise, 2004, 36, 674-688.

13. Kaminski, T.W., Buckley, B.D., Powers, M.E., Hubbard, T.J. and Ortiz, C., Effect of Strength and Proprioception Training on Eversion to Inversion Strength Ratios in Subjects with Unilateral Functional Ankle Instability, British Journal of Sports Medicine, 2003, 37, 410-415.

14. Engebretsen, A.H., Myklebust, G., Holme, I., Engebretsen, L. and Bahr, R., Prevention of Injuries Among Male Soccer Players: A Prospective, Randomized Intervention Study Targeting Players with Previous Injuries or Reduced Function, The American Journal of Sports Medicine, 2008, 36, 1052-1060.

15. Steffen, K., Myklebust, G., Olsen, OE., Holme, I. and Bahr, R., Preventing Injuries in Female Youth Football - A Cluster-Randomized Controlled Trial, Scandinavian Journal of Medicine and Science in Sports, 2008, $18,605-614$

16. Mazzetti, S., Kraemer, W., Volek, J., Duncan, N., Ratamess, N., Gómez, A., Newton, R.U., Häkkinen, K. and Fleck SJ., The Influence of Direct Supervision of Resistance Training on Strength Performance, Medicine and Science in Sports and Exercise, 2000, 32, 1175-1184.

17. Kraemer, W.J., Ratamess, N.A. and French, D.N., Resistance Training for Health and Performance, Current Sports Medicine Reports, 2002, 1, 165-171.

18. Pensgaard, A.M. and Roberts, G.C., Elite Athletes' Experiences of the Motivational Climate: The Coach Matters, Scandinavian Journal of Medicine and Science in Sports, 2002, 12, 54-59.

19. Coutts, A.J., Murphy, A.J. and Dascombe, B.J., Effect of Direct Supervision of a Strength Coach on Measures of Muscular Strength and Power in Young Rugby League Players, Journal of Strength and Conditioning Research, 2004, 18, 316-323.

20. Wisløff, U., Castagna, C., Helgerud, J., Jones, R. and Hoff J., Strong Correlation of Maximal Squat Strength with Sprint Performance and Vertical Jump Height in Elite Soccer Players, British Journal of Sports Medicine, 2004, 38, 285-288.

21. Granados, C., Izquierdo, M., Ibáñez, J., Ruesta, M. and Gorostiaga, E.M., Effects of an Entire Season on Physical Fitness in Elite Female Handball Players, Medicine and Science in Sports and Exercise, 2008, 40, 351-361.

22. Rousanoglou, E.N., Georgiadis, G.V. and Boudolos, K.D., Muscular Strength and Jumping Performance Relationships in Young Women Athletes, Journal of Strength and Conditioning Research, 2008, 22, 13751378.

23. Gustavsson, A., Neeter, C., Thomeé, P., Silbernagel, K.G., Augustsson, J., Thomeé, R. and Karlsson, J., A Test Battery for Evaluating Hop Performance in Patients with an ACL Injury and Patients Who Have Undergone ACL Reconstruction, Knee Surgery, Sports Traumatology, Arthroscopy, 2006, 14, 778-788.

24. Augustsson, S.R., Bersås, E., Magnusson, E., Sahlberg, M., Augustsson, J. and Svantesson U., Gender Differences and Reliability of Selected Physical Performance Tests in Young Women and Men, Advances in Physiotherapy, 2009, 11, 64-70.

25. Hickson, R.C., Hidaka, K. and Foster, C., Skeletal Muscle Fiber Type, Resistance Training, and StrengthRelated Performance, Medicine and Science in Sports and Exercise, 1994, 26, 593-58. 
26. Ekstrand, J. and Gillquist, J., Soccer Injuries and Their Mechanisms: A Prospective Study, Medicine and Science in Sports and Exercise, 1983, 15, 267-270.

27. Kraemer, W.J., Ratamess, N., Fry, A.C., Triplett-McBride, T., Koziris, LP., Bauer, J.A., Lynch, JM. and Fleck, SJ., Influence of Resistance Training Volume and Periodization on Physiological and Performance Adaptations in Collegiate Women Tennis Players, The American Journal of Sports Medicine, 2000, 28, 626633.

28. Augustsson, J., Esko, A., Thomeé, R. and Svantesson, U., Weight Training of the Thigh Muscles Using Closed vs. Open Kinetic Chain Exercises: A Comparison of Performance Enhancement, The Journal of Orthopaedic and Sports Physical Therapy, 1998, 27, 3-8.

29. Bell, N.S., Mangione, T.W., Hemenway, D., Amoroso, P.J. and Jones, B.H., High Injury Rates Among Female Army Trainees: A Function of Gender?, American Journal of Preventive Medicine, 2000, 18, 141146.

30. Stanganelli, L.C., Dourado, A.C., Oncken, P., Mançan, S. and da Costa, S.C., Adaptations on Jump Capacity in Brazilian Volleyball Players Prior to the Under-19 World Championship, Journal of Strength and Conditioning Research, 2008, 22, 741-749.

31. Kovacs, M.S., Pritchett, R., Wickwire, P.J., Green, J.M. and Bishop, P., Physical Performance Changes After Unsupervised Training During the Autumn/Spring Semester Break in Competitive Tennis Players, British Journal of Sports Medicine, 2007, 41, 705-710.

32. Campos, G.E.R., Luecke, T.J., Wendeln, H.K., Toma, K., Hagerman, F.C., Murray, T.F., Ragg, K.E., Ratamess, N.A., Kraemer, W.J. and Staron, R.S., Muscular Adaptations in Response to Three Different Resistance-Training Regimens: Specificity of Repetition Maximum Training Zones, European Journal of Applied Physiology, 2002, 88, 50-60.

33. Nuzzo, J.L., McBride, J.M., Cormie, P. and McCaulley, G.O., Relationship Between Countermovement Jump Performance and Multijoint Isometric and Dynamic Tests of Strength, Journal of Strength and Conditioning Research, 2008, 22, 699-707.

34. Ahtiainen, J.P., Pakarinen, A., Kraemer, W.J. and Häkkinen, K., Acute Hormonal and Neuromuscular Responses and Recovery to Forced vs Maximum Repetitions Multiple Resistance Exercises, International Journal of Sports Medicine, 2003, 24, 410-418.

35. Ahtiainen, J.P., Pakarinen, A., Kraemer, W.J. and Häkkinen, K., Acute Hormonal Responses to Heavy Resistance Exercise in Strength Athletes Versus Nonathletes, Canadian Journal of Applied Physiology, 2004, $29,527-543$.

36. Drinkwater, E.J., Lawton, T.W., Lindsell, R.P., Pyne, D.B., Hunt, P.H. and McKenna, M.J., Training Leading to Repetition Failure Enhances Bench Press Strength Gains in Elite Junior Athletes, Journal of Strength and Conditioning Research, 2005, 19, 382-388.

37. Goto, K., Nagasawa, M., Yanagisawa, O., Kizuka, T., Ishii, N. and Takamatsu, K., Muscular Adaptations to Combinations of High- and Low-Intensity Resistance Exercises, Journal of Strength and Conditioning Research, 2004, 18, 730-737.

38. Deschenes, M.R. and Kraemer, W.J., Performance and Physiologic Adaptations to Resistance Training, American Journal of Physical Medicine and Rehabilitation, 2002, 81, 3-16.

39. National Strength and Conditioning Association (NSCA) Strength \& Conditioning Professional Standards \& Guidelines, Available at http://www.nsca-lift.org/Publications/standards.shtml\#RECORDS, Accessed 28 November, 2008.

40. Bahr, R. and Bahr, I.A., Incidence of Acute Volleyball Injuries: A Prospective Cohort Study of Injury Mechanisms and Risk Factors, Scandinavian Journal of Medicine and Science in Sports, 1997, 7, 166-171.

41. Verhagen, E.A., Van der Beek, A.J., Bouter, L.M., Bahr, R.M. and Van Mechelen, W., A One Season Prospective Cohort Study of Volleyball Injuries, British Journal of Sports Medicine, 2004, 38, 477-481.

42. Aagaard, H. and Jorgensen, U., Injuries in Elite Volleyball, Scandinavian Journal of Medicine and Science in Sports, 1996, 6, 228-232. 
43. Batterham, A.M. and Hopkins, W.G., Making Meaningful Inferences About Magnitudes, International Journal of Sports Physiology and Performance, 2006, 1, 50-57. 
